\title{
Limulus psychophysics: Increment threshold
}

\author{
GERALD S. WASSERMAN \\ Purdue University, West Lafayette, Indiana 47907
}

\begin{abstract}
Prior temporal summation work had indicated that the sensory code for certain behaviors (in both Limulus and humans) can be understood if one suggests that the central nervous system analyzes the integral of the photoreceptor potential. An independent test of this suggestion is available because (in Limulus) the physiological increment threshold function obtained from the receptor potential integral is inflected, whereas that obtained from the initial transient peak of the receptor potential is not. The behavioral increment threshold function was measured in Limulus and found to be inflected. Fechner's scaling assumption (that equally detectable stimulus increments are mediated by sensory signals of equal size) was supported by the fact that a theoretical function, which was calculated from the receptor integral intensity-response function by using Fechner's scaling assumption, was able to fit the behavioral increment threshold function quite well. Furthermore, the variability of the behavioral data was proportional to the receptor integral variability. A seasonal effect was observed: Fall animals were more sensitive and had a higher criterion than winter animals. These findings permit the specification of the rerceptor potential that mediates this particular behavior at threshold: It has a steady-state amplitude of $7 \mathrm{mV}$ in winter and $16 \mathrm{mV}$ in fall. Taken in conjunction with the results of earlier temporal summation work indicating that the threshold receptor potential is $4.5 \mathrm{sec}$ long, this specification implies that at least some behaviors are mediated by large, long sensory signals, which have properties very different from those of small, short signals, particularly with regard to both linearity and their relative dependence on time vs. energy.
\end{abstract}

A sensory signal may be analyzed in a large number of ways. Any particular feature assessed by a particular analysis becomes a candidate sensory code whose candidacy can only be evaluated by determining whether or not a subsequent level of the nervous system can actually respond to that particular feature. Ultimately, the candidacy of any particular sensory code is evaluated by determining whether or not it is expressed in behavior (cf. Uttal, 1973).

A series of behavioral and physiological experiments on temporal summation (Kong \& Wasserman, 1978a, 1978b, 1978c; Wasserman, 1978; Wasserman \& Kong, 1974, 1975) suggested that the sensory code mediating the reflex tail response of Limulus, the horseshoe crab, was determined by the central nervous system's analysis of the integral of a peripheral sensory signal rather than by any momentary neural response feature. The suggestion was based on the presence of strongly nonlinear time-dependent effects in both physiology and behavior: Other temporal summation data (Hunter \& Sigler, 1940; Kahneman, 1966; Kaswan \& Young, 1963) indicate that human observers also behave, in certain identification tasks, in ways that suggest that the human sensory code also

This work was supported by NIH Grant NS 12,693 and NSF Grant BNS 78-02359. I would like to thank Judy Borowitz for her assistance and R. B. Barlow, Jr., D. R. Pepperberg, W. R. Uttal, and an anonymous referee for helpful comments on earlier drafts. Requests for reprints may be directed to the author at the Sensory Coding Laboratory, Department of Psychological Sciences, Purdue University, West Lafayette, Indiana 47907. sometimes involves the integral of sensory signals, because corresponding time-dependent nonlinearities were found in these cases as well.

This evidence comes mainly from investigations of the time dimension. Obviously, one would want to explore other dimensions of sensation in the same detail before accepting this suggestion. That was the purpose of the present investigation of Limulus sensory behavior, which examined the increment threshold in Limulus. This problem was chosen because physiological data exist that differentiate incremental responses mediated by the receptor integral from other possible codes: Following an earlier suggestion of duality in the Limulus eye made by Wulff (1950), which was later reinforced by Dowling (1968), Barlow and his co-workers have shown that a clear-cut difference exists between the increment threshold mediated by the peak of the initial receptor transient as opposed to the increment threshold obtained by integrating the receptor response for a long period of time (Barlow \& Kaplan, 1977; Bayer \& Barlow, 1978; Kaplan \& Barlow, 1975). The peak increment threshold is a linear function of log background intensity over a wide range. The integral increment threshold, in contrast, exhibits two clearly defined segments as a function of background intensity with an inflection at the junction of the two segments. This difference is most marked during the daytime (Barlow, Boyanowski, \& Brachman, 1977). The cause of this difference in the form of the increment threshold functions is an underlying difference in the form of 
the intensity-response functions: For the peak, the intensity-response function is not inflected; for the integral, it is. If the conclusion about Limulus psychobiology drawn from studying temporal summation (Wasserman, 1978) is correct, and if the visually guided behavior of Limulus is really mediated by the central nervous system's analysis of the integral of receptor responses, then the behavioral increment threshold function should also exhibit two segments joined by an inflection.

\section{METHOD}

The psychophysical technique used was the same as that described previously, unless otherwise specified (Wasserman, 1973, 1975, 1976, 1978; Wasserman \& Patton, 1970). Briefly, animals were clamped into a harness that permitted only tail movements. A string was attached to the tail and to a sensitive potentiometer as well so that movements of the tail produced analog voltage signals. A gated analog detector produced a relay closure when the position of the tail changed by $6 \mathrm{~mm}$ from its position at light onset. The light stimulus was delivered to the ventral eye because it is prepotent for this behavior (Wasserman, 1973).

\section{Apparatus}

The apparatus used in this work was the same as that described earlier, except as noted below. The light stimulus was drawn from a xenon arc lamp and filtered by a heat filter. In order to obtain the intensities necessary to study the increment threshold over a wide range, the xenon arc used in the present study had a 1,000-W lamp (Hanovia 982C) mounted in a blower-cooled housing (Shoeffel LH-152). The lamp current was monitored and adjusted manually as needed. The intensity of the light was adjusted by a pair of counterbalanced Inconel neutral density wedges. A new set of wedges (Kodak A6040) was installed in order to obtain a wider operating range. With these new wedges, the test-flash intensity could be varied over a range of $6 \mathrm{log}$ units. In order to maximize the amount of light available, a larger ultraviolet-enhanced fiber optic light guide (American Optical ULGM-5-48) was used to convey the light from the stimulator to the animal. This new light guide was $.64 \mathrm{~cm}$ in diameter and was located, as before, about 2 to $3 \mathrm{~cm}$ from the ventral eye. The exact distance was determined by the size and shape of the subject's ventral surface. The test flash was gated so that its maximum duration was $10 \mathrm{sec}$, but responses with a latency less than $10 \mathrm{sec}$ terminated the test flash. The light used for the background stimulus was drawn by means of a beam splitter from the same optical channel as the test stimulus and was recombined with the test stimulus by another beam splitter. The intensity of the background light was adjusted by inserting fixed Inconel neutral density filters (Bausch \& Lomb 33-86 series). The background intensities were chosen in the following manner: The most intense background was set by the intensity of the adapting beam with no neutral density filters inserted. This maximal background was approximately $1.6 \mathrm{log}$ units lower than the intensity of the most intense test flash because the double attenuation of the two beam splitters favored the test flash. Light shields and baffles were added to the optical system until the background intensity was reduced to a level that was only four times the noise level of our radiometer. This lowest background intensity was more than 6 log units below the maximum background and represented a mid-scotopic level. This gave a 7.9-log-unit difference between the weakest background and most intense test flash.

The stimuli were calibrated radiometrically with a Tektronix J-16 digital photometer-radiometer equipped with a J-6512 radiometric probe. This Tektronix radiometer had been previously compared with two other radiometers used by colleagues. At $500 \mathrm{~nm}$, the Tektronix readings were $.08 \log$ units lower than those given by a PIN 5DT photodiode and .15 log units lower than those given by an EGG radiometer. ${ }^{1}$ Such small differences are well within the normal tolerances of these devices and are insignificant in the present context.

Since a comparison of the results of this experiment with the work done by Bayer and Barlow (1978) on single cells required a conversion of radiance measures to quantal measures, the stimuli used in the present experiment were also calibrated spectroradiometrically. A J-6514 probe (whose spectral sensitivity was provided by Tektronix) was used with Zeiss (Jena) interference filters that had been individually calibrated in a Beckman DK-2 recording spectrophotometer. The peak wavelengths of these filters were nominally spaced at $25-\mathrm{nm}$ intervals from 350 to $700 \mathrm{~nm}$. These filters were placed in a collimated portion of the light beam, and they contained dye filters that blocked sidebands. The validity of the spectroradiometric curve was checked by comparing its integral from 350 to $700 \mathrm{~nm}$ with the net radiance measured with the $\mathrm{J}-6512$ radiometric probe; these two values differed by less than $.1 \mathrm{log}$ unit. This high agreement must be fortuitous since the spectroradiometric calibration did not assess the small amount of infrared light that passed the heat filter, while the J-6512 probe is relatively insensitive in the ultraviolet. Apparently, these two factors cancelled each other.

The spectroradiometric data alone are not enough to compare our behavioral stimuli with the physiological stimuli used by Bayer and Barlow (1978), because the optics involved in the two experiments differed. They recorded from excised cells taken from the ventral eye of Limulus. On the other hand, in our behavioral investigations, the ventral eye is covered in situ by a cuticle that has a strong wavelength-dependent absorption (Wasserman, 1976); it absorbs very strongly at short wavelengths and is visibly yellow or brown. Therefore, the spectroradiometric calibrations of the behavioral stimuli were adjusted to allow for the spectral absorption of the ventral eye cuticle; the details of these calculations were given earlier (Wasserman, 1976). Figure 1 incorporates this adjustment and shows the quanta incident per second per nanometer per receptor unit as a function of wavelength when the maximum intensity test flash was applied. This test flash had an intensity, when incident on the cuticle, of $5.5 \log \mathrm{mW} / \mathrm{m}^{2}$. The spectrum of the stimulus used by Bayer and Barlow (as well as by Barlow \& Kaplan, 1977) is also shown in Figure 1 with an arbitrary vertical location. ${ }^{2}$ These two spectra are generally similar. However, the behavioral stimulus contained some ultraviolet light, while Bayer and Barlow's stimulus contained virtually none. The wavelength in-

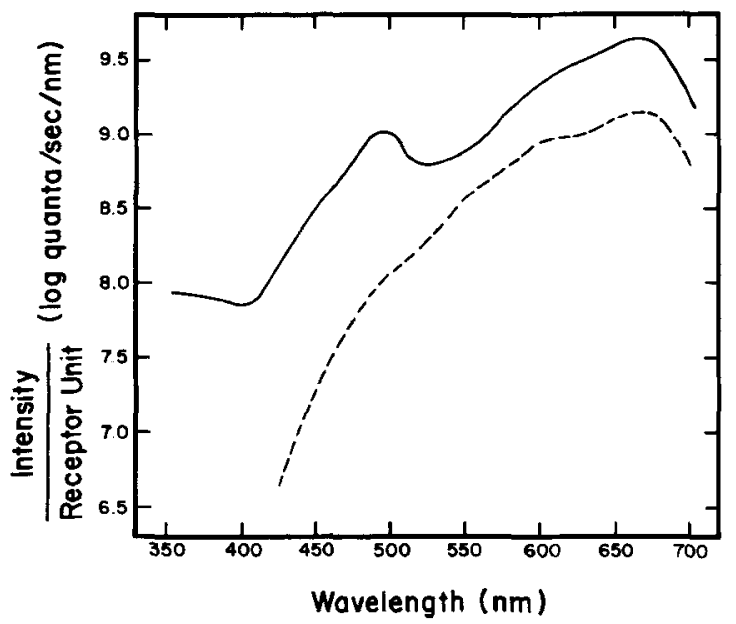

Figure 1. Stimulus spectral distribution given as the quanta incident on each receptor per second per nanometer (nm) as a function of wavelength (in $\mathrm{nm}$ ). Solid line describes the most intense stimulus used in the present work; dashed line, the stimulus used by Bayer and Barlow (1978). Their spectrum has an arbitrary vertical location. 
tegral of the spectrum in Figure 1 gives the number of incident quanta per second per receptor unit. The most intense behavioral stimulus (i.e., at $5.5 \log \mathrm{mW}, \mathrm{m}^{2}$ ) represented an incident flux of $5 \times 10^{\prime \prime}$ quanta/sec/receptor. For comparison, the quantal flux of this most intense stimulus when incident on the cuticle was $8 \times 10^{20}$ quanta $/ \mathrm{m}^{2} / \mathrm{sec}$. The shape of Bayer and Barlow's spectrum is determined by two factors: (1) the general decline at short wavelengths characteristic of tungsten lamps, and (2) the further attenuation at short wavelengths produced by glass optics. The shape of the spectrum of the stimulus used in the present experiment is determined by four factors: (1) the general decline at short wavelengths produced by the yellow cuticle, (2) the sharp decline at long wavelengths produced by the heat filter, (3) the peak near $490 \mathrm{~nm}$ produced by lines in the xenon spectrum, and (4) the dip near $400 \mathrm{~nm}$ produced by the antireflection coating on our lenses.

\begin{abstract}
Animals
Gulf Specimen Company of Panacea, Florida, provided the animals which were kept in a room whose lights went on at 8 a.m. and off at 8 p.m. All testing was done between 12:30 p.m. and 4:30 p.m. As in previous work, the animals used in this study were first screened for the presence of the reflex tail response. The same screening criterion was used-namely, that after 3 days of testing, the animals had to respond to a suprathreshold light at a level of $75 \%$ or better. Single check sessions were used later with the same criterion. The dimmest background and the most intense test flash were used for screening. This test flash was approximately $1.25 \mathrm{log}$ units more intense than the most intense test flash used in our previous behavioral research. The sensitivity of the animals actually studied is affected by the intensity of the test flash used for screening: the less intense the screening test flash, the more sensitive the animals that pass the screen. As a result, few animals were rejected in the present experiment by the initial screening test: Of the 13 animals screened, 11 met criterion before the experiment. Of these 11 animals, 10 met criterion after at least one replication and hence contributed to the data. Of these animals, three completed one replication of the experiment, while four completed two, one completed three, one completed four, and two completed five. Thus, the data in this experiment come from 10 of 13 animals, or $77 \%$ of the population. In contrast, in earlier experiments with weaker screening lights, fewer animals contributed. In Wasserman (1975), 8 of 23 contributed; in Wasserman (1976), 6 of 24 contributed; and in Wasserman (1978), 14 of 39 contributed. In these earlier experiments, only $33 \%$ of the population passed criterion, on the average.
\end{abstract}

\section{Procedure}

Since individual animals can only be used for a limited number of sessions before they cease to respond, it is necessary to use an experimental design that monitors and controls for possible response potency changes. The following design was used: Each session used only one background intensity, and the animal was adapted to that background for $15 \mathrm{~min}$ before test flashes were presented. An ascending series of test-flash intensities has generally been presented because suprathreshold lights were expected to depress the response to threshold lights; the use of descending intensities would therefore affect the results to a variable degree that would depend upon the experimenter's arbitrary choice of the starting intensity. On the first trial, the neutral density wedges have generally been set at their maximum attenuation. On each succeeding trial, a stepping motor increased the test-flash intensity in the present use by approximately $.6 \mathrm{log}$ units. The present experiment included a check on this assumption: The first trial in any session presented the most intense test flash. The second trial presented the weakest test flash. Successively more intense test flashes were presented until the session ended on the 11 th trial with a repetition of the most intense test flash. Intertrial interval was $2 \mathrm{~min}$. No significant difference was found in the responses to the first and last presentation of the most intense test flash; the mean difference in response frequency was $1.9 \%(\mathrm{SE}=4.6 \%)$. However, the first and most intense test flash did depress the response to the second and weakest test flash; all of the latter responses were indiscriminable from the spontaneous response level.

Seven background intensities were used altogether. Each session used one of the seven background intensities presented in a counterbalanced order. The seven sessions in any one order constituted one complete replication of all of the test and adapting intensities used in the experiment. Fourteen different orders were necessary to counterbalance completely the design across animals. Following the seven sessions for any given order, a check session was run with the same conditions that were used in the screening sessions (namely, the most intense test flash presented against the least intense background). If the performance in any given check session indicated that an animal was no longer responding at criterion, the entire set of observations from the seven sessions of that particular replication was discarded. Responsive animals continued to be tested until they stopped responding.

\section{Seasonal Effect}

The experiment was begun in mid-September, and 14 replications were completed by mid-November. The entire experiment was repeated a second time, and 14 more replications were run in the same counterbalanced order from mid-November to midFebruary. The results of the two experiments were generally comparable. However, results from other work that covered a whole year indicated that a large seasonal effect exists in Limulus, with summer animals being approximately $2 \log$ units more sensitive than winter animals (Wasserman, Note 1). Because of this finding, the results of the present two experiments were analyzed separately.

\section{RESULTS}

The data consist of a matrix of response frequencies evoked by test flashes of varying intensity superimposed on backgrounds of varying intensity. At any given combination of test and background, 14 trials were presented in each experiment. Some trials were preceded by spontaneous movements starting before test-flash onset. These spontaneous responses were recorded separately by the same gated analog detector used to measure the light-evoked responses. All such trials were discarded. Hence, the response frequencies are the number of trials on which a criterion tail movement occurred, leaving out trials affected by spontaneous responses, divided by 14 minus the number of spontaneous responses. Figures 2 and 3 show the response frequencies for the fall and winter experiments, respectively, as a function of testflash intensity with background intensity as a parrameter. In order to present these data clearly, the results obtained with each successive background intensity have been shifted vertically by $20 \%$, and separate shifted response scales are shown in Figures 2 and 3 for each of the background intensities. Omitted from these figures are all cases that produced response frequencies that were less than $10 \%$. Such cases were considered to be insufficiently discriminable from spontaneous responses. Psychometric functions, in the form of least squares best-fitting cumulative normal curves, were calculated at each background intensity by transforming the test-flash 
Log Test Flosh Intensity $\left(\mathrm{mW} / \mathrm{m}^{2}\right)$

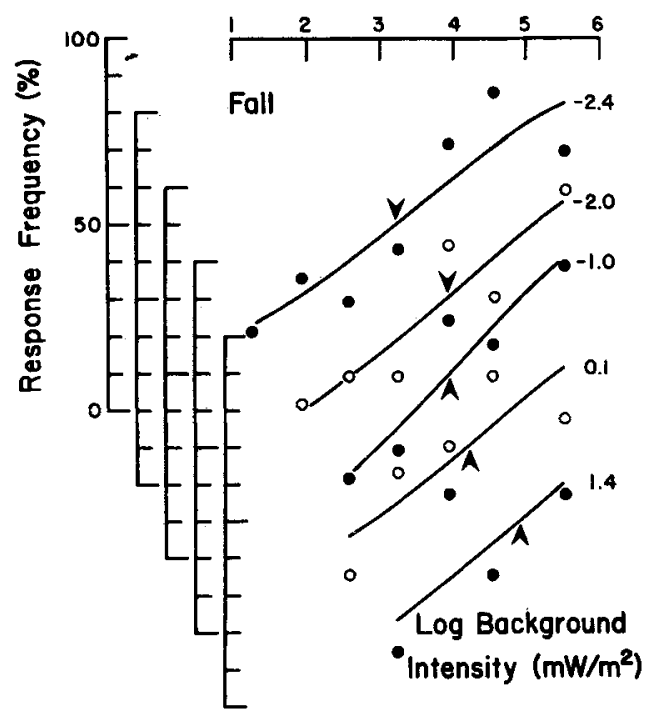

Figure 2. Response frequency (in percent) as a function of testflash intensity (in $\log \mathrm{mW} / \mathrm{m}^{2}$ ) for the fall experiment. For clarity, the frequencies for each background have been shifted vertically by $20 \%$ and a separate scale given for each. Curves are the least squares best fitting probit functions; markers on each curve indicate the mean threshoid.

\section{Log Test Flosh Intensity $\left(\mathrm{mW} / \mathrm{m}^{2}\right)$}

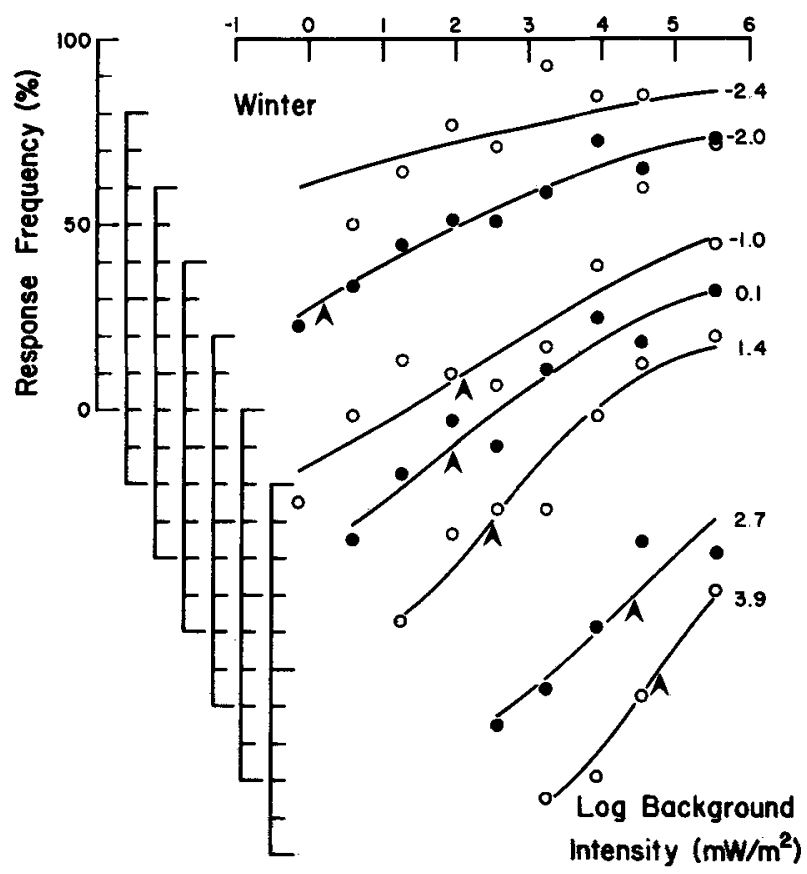

Figure 3. Data from the winter experiment composed in the same manner as in Figure 2.

response frequencies into probit values; the fitted functions are also shown in Figures 2 and 3. These functions are the cumulative integrals of underlying normal distributions of the thresholds. Hence, the mean thresholds are given by the $50 \%$ points on the psychometric functions, and the standard deviations of the thresholds are the reciprocals of the probit slopes (Finney, 1971).

Increasing background intensity translates the psychometric functions horizontally along the test-flash intensity axis, so the mean threshold intensity changes with background adaption. To facilitate examination of this threshold shift, markers have been provided in Figures 2 and 3 that indicate the mean test-flash threshold obtained at each background intensity. (Threshold for the -2.4-log-unit background in the winter was below the lowest test-flash intensity and had to be estimated by extrapolation.) An orderly increment threshold effect is visible; the higher the background intensity, the higher the mean threshold. In addition, a very pronounced effect of background intensity on the slope of the psychometric function is also evident in the winter. At the lowest background intensity, the slope is shallowest; the slope increases substantially with increasing background intensity. At the highest background intensity, the slope (in probit space) of the psychometric function is about five times as great as the slope of the curve obtained at the lowest background intensity.

The functional relation between background intensity and test-flash threshold is illustrated in Figure 4, which also shows curves representing the theoretical predictions that stem from the integral coding hypothesis. The error bars in Figure 4 are standard errors taken as the standard deviations of the data in Figures 2 and 3, divided by the square root of the number of observations. The best-fitting prediction that could be obtained from the notion that it is the peak of the transient response to the test flash which mediates threshold would be that log threshold is a linear function of $\log$ background intensity. The slope of this linear function in the fall would be .38, and such a linear fit would pass close to or through most of the error bars. However, in the winter, the slope would be .91, and the fit of the peak prediction to the winter behavioral data would be very unimpressive because the error of estimate is small enough to rule out the possibility that the differences between the peak theory and the winter data are random variates. Using the best-fitting lines to fit the data plotted in Figure 4 would actually overstate the agreement between the peak theory and the data, because the slope of the straight line in Kaplan and Barlow's (1975) peak recordings was .7. Thus, the winter slope would be too steep, and the fall slope too shallow.

Inspection alone indicates that the data from both experiments will be best described by an inflected increment threshold function. This qualitative conclusion from inspection can be tested quantitatively as well. Figure 4 compares the behavioral data with calculations generated from the hypothesis that it is the integral of the response that determines behavior. As 


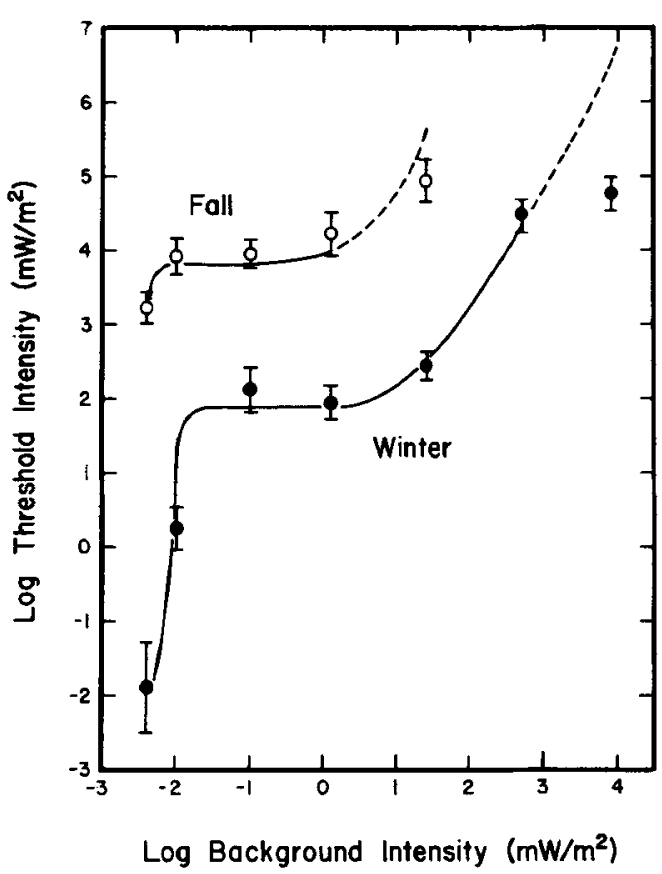

Figure 4. Test-flash threshold (in $\log \mathrm{mW} / \mathrm{m}^{2}$ ) as a function of background (in $\log \mathrm{mW} / \mathrm{m}^{2}$ ) for the fall (open circles) and winter (filled circles). Vertical bars indicate \pm 1 standard error. The solid curves represent values predicted from the response integral theory described in the text. The dashed lines represent portions of these predictions based on extrapolation.

Kaplan and Barlow (1975) noted, these values can be obtained from the steady-state intensity-response function by direct calculation if one uses Fechner's scaling assumption (namely, that equally discriminable light increments correspond to equal changes in the sensory signal). Such calculations were done for the behavioral results using Bayer and Barlow's (1978) integrated intensity-response data from the ventral eye. ${ }^{3}$ Since the behavioral data of the present experiment were mediated by the ventral eye, physiological data obtained from the ventral eye by Bayer and Barlow (1978) were used here for comparison whenever possible. The solid portions of the curves in Figure 4 represent direct calculations. The dashed portions are based on an extrapolation of Bayer and Barlow's data. Because the thresholds at the highest backgrounds fall below the dashed line in both fall and winter, it is possible this extrapolation is incorrect.

These quantitative results further support the notion that it is the integrated neural response, and not the transient, that mediates this behavior. The size of the receptor potential increment at behavioral threshold is given directly by the calculation described in detail in Footnote 3. In the fall, it is $16 \mathrm{mV}$; in the winter, it is $7 \mathrm{mV}$. Both signal levels are well into the nonlinear range of this sensory system. ${ }^{4}$ In addition, the same calculation gives the sensitivity during the two seasons; the fall animals are $.5 \log$ units more sensitive than the winter animals, which is consistent with results found in a more extensive seasonal experiment (Wasserman, Note 1). Sensitivity cannot be evaluated simply by examining the data in Figure 4, because the data give a paradoxical appearance: the winter animals are more "sensitive" when reading along the test-flash threshold axis, but the fall animals are more "sensitive" when reading along the background axis. The calculation described in Footnote 3 resolves this paradox into two components-an increase in true sensitivity in the fall and a lower criterion or bias in the winter. This distinction is similar to the distinction made in signal detection theory (Green \& Swets, 1974). The spontaneous frequencies differed in a direction consistent with this interpretation: In the fall, they had a frequency of $2.2 \%$; in the winter, $4.0 \%[\mathrm{t}(6)=3.11$, $p<.05]$. Thus, when the animals' criterion was reduced by a factor of approximately 2 (from 16 to $7 \mathrm{mV}$ ), the spontaneous response level increased by a factor that was comparable in magnitude. But, to be fully consistent with this interpretation, the spontaneous responses should be systematically related to the background intensity. As Table 1 shows, this is not the case. In the fall, the spontaneous responses decline by $.16 \%$ for every log unit increase, while in the winter, they increase at a rate of $.19 \%$ per log unit and, on the average, there is little $(.03 \%$ per log unit) change. The most likely basis for this discrepancy is that there are two kinds of spontaneous movement. One kind can be directly observed; it consists of a prolonged series of movements of legs, tail, and carapace, which appear to represent an (occasionally successful) attempt to escape from the apparatus. This escape behavior would not be expected to be related to light intensity. By hypothesis from the fall-winter difference, a second type of spontaneous movement should exist; it should consist of a phasic downward tail movement with a form similar to responses evoked by the test flashes. Spontaneous responses with this latter character have been observed visually. However, the analog detector that collects quantitative data would not discriminate between these two types, so no quantitative record of their relative frequency is

Table 1

Spontaneous Response Frequencies as a Function of Background Intensity

\begin{tabular}{cccc}
\hline $\begin{array}{c}\text { Background } \\
\left(\log \mathrm{mW} / \mathrm{m}^{2}\right)\end{array}$ & Fall & Winter & Mean \\
\hline 3.9 & 2.1 & 3.6 & 2.9 \\
2.7 & 2.1 & 5.0 & 3.6 \\
1.4 & .7 & 5.0 & 2.9 \\
.1 & 2.1 & 4.3 & 3.2 \\
-1.0 & 3.6 & 3.6 & 3.6 \\
-2.0 & 2.9 & 3.6 & 3.2 \\
-2.4 & 2.1 & 2.9 & 2.5 \\
Mean & 2.2 & 4.0 & 3.1 \\
\hline
\end{tabular}


available. If there really are two types of spontaneous activity, the random escape activity would be expected to obscure the effect of the systematic light-related activity in small sample statistics, but larger samples should reduce this obscuration. This seems to be the case for the two analyses made from the data of Table 1; the seasonal comparison is based on a single partition of the data, while the background trend comparisons depend on finer, and hence smaller, partitions.

The variability of the behavioral data can also be quantitatively related to physiology if one uses an integrated response measure. Kaplan and Barlow (1975) reported the standard deviation of the steady-state neural response as a function of stimulus intensity (their Figure 11). This physiological information can be quantitatively related to the variability of the behavioral data by a calculation that simply assumes that the behavioral threshold variability (VB) is proportional to the variability in the physiological response (VP) plus the between-animals variability (VA) or that VB = kVP + VA. The value of the constant of proportionality, $k$, has no significance since the physiological data were reported in normalized form. After accounting for the sensitivity, ${ }^{5}$ there are two free parameters in this calculation (the constant of proportionality and the between-animals intercept). The standard deviation of the behavioral data is equal to the reciprocal of the slopes of the probit functions that were shown in Figures 2 and 3; the higher the slope, the lower the standard deviation. Inserting the physiological and behavioral values into the expression given above then yields a least squares fit for $k$ and VA. Once these parameters have been obtained, it is a straightforward matter to calculate the behavioral variability that would be expected to result from a given physiological variability. Figure 5 shows the behavioral variability calculated from the physiological variability as a function of the behavioral variability that was actually measured. The variabilities are expressed in terms of the probit slopes. The winter results indicate that the physiological variance gives a good account of the behavioral variability when the between-animals variability is taken into account. Similar slope changes have also been seen in human psychophysical studies of the increment threshold (Mueller, 1951); the changes in Mueller's psychometric functions taken from human observers can be interpreted in the same fashion as the Limulus datanamely, as expressions of variability in sensory signal strength. But the fall results have a very restricted range. In addition, the probit slopes for the fall data are lower (and hence the standard deviations are higher) than corresponding points in the winter. The explanation for these differences comes from the year-long seasonal study mentioned earlier (Wasserman, Note 3): Limulus exhibits a bimodal distribution of sensitivities. In summer, most of the animals are in

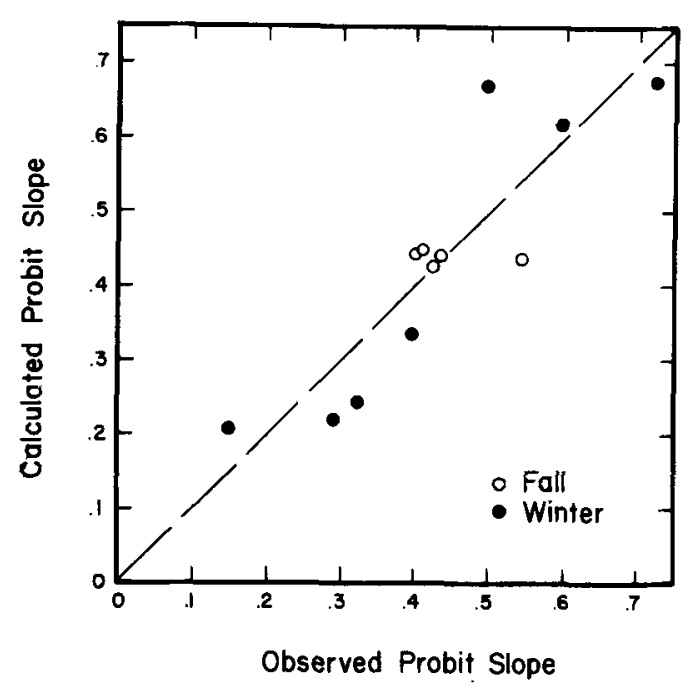

Figure 5. Probit slopes calculated from Kaplan and Barlow's (1975) single cell variance data by the method described in the text as a function of the probit slopes actually observed in Figures 2 and 3 of the present behavioral experiment.

the more sensitive state; in winter, most of the animals are in the less sensitive state. In fall and spring, however, animals are more equally distributed between the two modal states. Hence, the betweenanimals variability should be much greater in fall than in winter. And the between-animals variability calculated from the data of the present experiment was actually 1.6 times greater in fall than in winter. This inflation of the between-animals variability in fall obscured the relation between physiology and behavior that is so clear in the winter data.

The fact that the behavioral variability can be predicted from the physiological data when betweenanimals variability is taken into account means that each point on the psychometric functions can be predicted from physiology. Therefore, the theoretical analysis given in Figure 4 is in no important way sensitive to the fact that the behavioral means (at the 50\% points on the psychometric functions) were compared with the physiological means. One could just as well have used the means-plus-one standard deviation (at the $84.1 \%$ points) for both physiology and behavior and obtained an equivalent fit, even though such calculations would have been more tedious.

\section{DISCUSSION}

The primary conclusion is that this particular visually guided behavior in Limulus is mediated by relatively large receptor signals that are integrated by the central nervous system over a long period of time. This conclusion is qualitatively the same as the conclusion reached from an earlier temporal summation experiment (Wasserman, 1978). The two experiments taken together give an exact quantitative account of 
the signal in a Limulus photoreceptor at behavioral threshold; the signal is $4.5 \mathrm{sec}$ long and has a steadystate amplitude of $16 \mathrm{mV}$ in fall and $7 \mathrm{mV}$ in winter. The upper trace of Figure 6 shows a representation of the receptor potential that would produce a behavior that is just at threshold in the fall on this interpretation. This representation has been adapted from Barlow and Kaplan's (1977) Figure 7, which provides a receptor potential that matches the fall behavioral threshold parameters. (In adapting Barlow and Kaplan's results, the quantum fluctuations that are prominent in single cells were averaged out because behavior would clearly be mediated by the average signal coming from a population of receptors.) The lower trace of Figure 6 shows the receptor signal that would be just detectable by a physiologist, using sensitive electronic instrumentation. The physiologist's threshold signal occurs when a single quantum is absorbed, producing a potential variously known as a quantum bump, a miniature potential, or a slow potential fluctuation. When these two threshold level signals are represented on the same time and gain scales, the physiologist's threshold signal is hardly noticeable. The winter threshold signal would, of course, be smaller. Its plateau would only reach $44 \%$ of the amplitude of the fall signal. Its initial transient would be even more sharply reduced and would probably reach a value only a quarter of the corresponding value reached by the fall signal. Nevertheless, the two behavioral threshold signals would be far more similar to each other than either is to the physiologist's threshold, and both are well into the nonlinear range (cf. Footnote 3).

\section{Threshold Receptor Potentials}

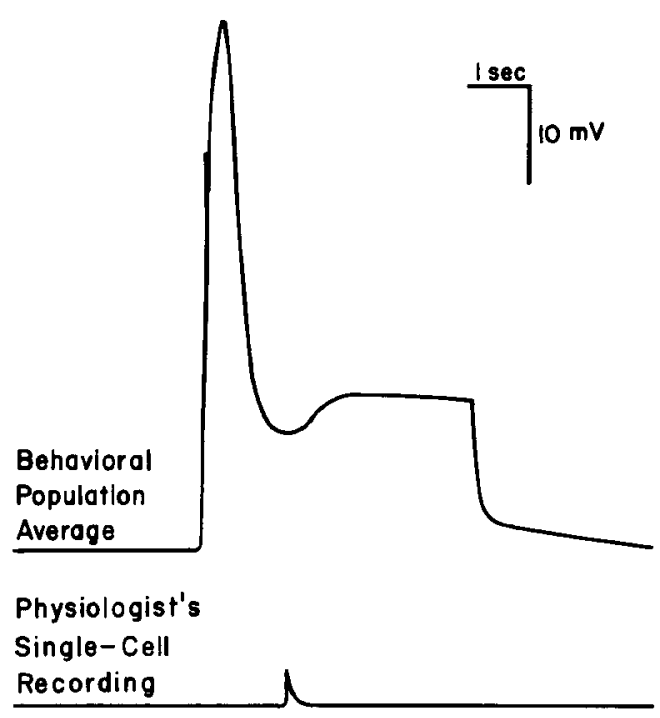

Figure 6. Receptor potentials at behavioral and physiological threshold. The behavioral threshold potential represents the population average in the fall; it has been adapted with smoothing from Barlow and Kaplan (1977). The physiologist's threshold potential is given as a single quantum potential.
This does not imply that all threshold behaviors in all organisms (or even in Limulus) are necessarily mediated by such large and prolonged receptor signals. Clearly, when the task and the conditions of the experiment are arranged appropriately, much smaller sensory signals can mediate behavior at threshold. And Fain, Granda, and Maxwell (1977) reported a threshold receptor potential in the turtle that is more than three orders of magnitude smaller in amplitude and probably (although there is no direct evidence) two orders of magnitude smaller in duration. Nevertheless, the behavioral sensitivities are fairly comparable, for Limulus achieves its high sensitivity through extensive temporal summation, while the turtle probably achieves its high sensitivity through extensive spatial summation. The level of sensitivity that the present data represent is worth emphasizing by providing some benchmarks: $6 \mathrm{log} \mathrm{mW} / \mathrm{m}^{2}$ is approximately noon sunlight, 0 is approximately human photopic threshold, and -4 is approximately human scotopic threshold. All of the data from the present experiment are consistent with the notion that the Limulus ventral eye has already entered the region of nonlinear response compression at intensities that are comparable with the human scotopic threshold (cf. Figure 7; from approximately $-4 \log \mathrm{mW} / \mathrm{m}^{2}$ to approximately $-2 \log \mathrm{mW} / \mathrm{m}^{2}$, the response is a logarithmic function of intensity).

Therefore, it is important to attend to the general situation in attempting to build a complete psychobiological theory of sensory coding. Small brief sensory signals have very different properties than do large prolonged signals: The former are highly linear, while the latter are profoundly nonlinear (see review by MacLeod, 1978). If a general theory of behavior were erected on the presupposition that all threshold level behaviors necessarily are mediated by linear or quasilinear sensory signals, then that theory would generally be in error even if it were successful in a limited domain. Nonlinearities are apt to be particularly prominent in form perception and identification situations in which the demands of the task make it impossible to achieve high sensitivity by spatial summation, forcing the subject to rely more on temporal summation. And human psychophysics does show that form perception involves lengthier temporal summation (Hunter \& Sigler, 1940; Kahneman \& Norman, 1964). Another related difference is that the integral of the receptor potential exhibits strong time dependencies, whereas the initial peak is energy dependent (cf. Kong \& Wasserman, 1978a). Behaviors mediated by the integral code should therefore be strongly time dependent.

The difference between time- and energy-dependent behaviors has often been used to implicate different underlying mechanisms. For example, in the area of backward visual masking, energy-dependent effects have often been taken as evidence for a peripheral integrative mechanism, while time-dependent effects 
have often been taken as evidence for a central interruptive mechanism. A review of the masking problem is given by Felsten and Wasserman (1980). The present data support their suggestion that behavioral differences in visual masking are expressions of coding differences.

Yet another implication of the present results is one that has already been pointed out by Kaplan and Barlow (1975). Behavioral increment threshold data have often been used in an attempt to parse sensory systems into components; these components are generally attributed to differences between nerve cells. A particularly striking example of this kind of analysis is that of fered by Stiles (1959), who analyzed the mechanisms underlying color vision into five components as a result of his studies of the increment thresholds of differently colored lights presented on differently colored backgrounds. Stiles' results have always been a problem: Although he came very close to measuring accurately the spectra of the three primate cone types, the fact that his method yielded five components, three with very similar spectra, has been difficult to understand. But, as Barlow and Kaplan (1977) pointed out, a complex increment threshold function can be obtained within a single photoreceptor with a single spectrum. The occurrence of the same increment threshold pattern in the present behavioral results supports and lends generality to Barlow and Kaplan's conclusion. Furthermore, one can suggest that the three Stiles components that have similar spectra are probably primarily from one cone (the $S$ cone), and one can further suggest that this cone differs from the other two in that its signals are integrated over a longer period.

It is interesting that Fechner's scaling assumption, which has usually been a pure assumption without converging evidence to support it, is shown here directly to explain Limulus behavior. In this useful model organism, one can carefully control the stimulus, measure the sensory signal, and monitor the behavior. The fact that Fechner's scaling assumption ties these three levels together in a satisfactory way confirms and supports the validity of Fechner's assumption. Moreover, Fechner's scaling assumption is shown here to be appropriate in a context in which Weber's law is certainly not valid. As Figure 4 shows, the Weber fraction varies considerably as the background changes. In winter, it ranges from a low of about 3 to a high of about 1,300. Luce and Edwards (1958) presented a theoretical demonstration that Fechner's scaling assumption could be dissociated from the particular empirical context from which it was generated. The present data support their analysis and emphasize the importance of dissociating Fechner's scaling principle from Weber's empirical finding. Fechner's principle only leads to a logarithmic psychophysical function for those particular restricted sit- uations in which Weber's law is valid. This interpretation predicts that a magnitude estimation experiment (Marks, 1974) that asked observers to adjust an incremental light flash so that it was a criterion amount brighter than a background light would yield a psychophysical function that follows a power law. Fechner's principle should thereby yield Stevens' law.

\section{REFERENCE NOTES}

1. Wasserman, G. S. Bistable daily and seasonal changes in Limulus visual sensitivity. Manuscript in preparation, 1981.

2. Clack, J. W., \& Pepperberg, D. R. Personal communication, August 1977.

3. Barlow, R. B., Jr. Personal communication, March 1978.

\section{REFERENCES}

Barlow, R. B., Boyanowski, S. J., \& Brachman, M. L. Efferent optic nerve fibers mediate circadian rhythms in the Limulus eye. Science, 1977, 197, 86-89.

Barlow, R. B., \& Kaplan, E. Properties of visual cells in the lateral eye of Limulus in situ: Intracellular recordings. Journal of General Physiology, 1977, 69, 203-220.

Bayer, D. S., \& Barlow, R. B. Limulus ventral eye: Physiological properties of photoreceptor cells in an organ culture medium. Journal of General Physiology, 1978, 72, 539-563.

Dowling, J. E. Discrete potentials in the dark-adapted eye of Limulus. Nature, 1968, 217, 28-31.

Fain, G. L., Granda, A. M., \& Maxwell, J. H. Voltage signal of photoreceptors at visual threshold. Nature, 1977, 265, 181-183.

Felsten, G., \& Wasserman, G. S. Visual masking: Mechanisms and theories, 1980. Psychological Bulletin, 1980, 88, 329-353.

Finney, D. J. Probit analysis. Cambridge: Cambridge University Press, 1971.

Green, D. M., \& Swets, J. A. Signal detection theory and psychophysics. Huntington, N.Y: Krieger, 1974.

Hunter, W. A., \& Sigler, M. The span of visual discrimination as a function of time and intensity of stimulation. Journal of Experimental Psychology, 1940, 26, 160-179.

Kahneman, D. Time-intensity reciprocity under various conditions of adaptation and backward masking. Journal of Experimental Psychology, 1966, 71, 543-549.

Kahneman, D., \& Norman, J. The time-intensity relation in visual perception as a function of the observer's task. Journal of Experimental Psychology, 1964, 68, 215-220.

Kaplan, E., \& Barlow, R. B. Properties of visual cells in the lateral eye of Limulus in situ: Extracellular recordings. Journal of General Physiology, 1975, 66, 303-326.

Kaswan, J., \& Young, S. Stimulus exposure time, brightness, and spatial factors as determinants of visual perception. Journal of Experimental Psychology, 1963, 65, 113-123.

Kong, K.-L., \& Wasserman, G. S. Changing response measures alters temporal summation in the receptor and spike potentials of the Limulus lateral eye. Sensory Processes, 1978, 2, 21-31. (a)

Kong, K.-L., \& Wasserman, G. S. Temporal summation in the receptor potential of the Limulus lateral eye: Comparison between retinula and eccentric cells. Sensory Processes, 1978, 2, 9-20. (b)

Kong, K.-L., \& Wasserman, G. S. Two linear rules relate the latencies of visual responses to their critical durations. Sensory Processes, 1978, 2, 1-8. (c)

Luce, R. D., \& EDwards, W. The derivation of subjective scales from just noticeable differences. Psychological Review, $1958,65,222-237$.

MacLeOD, D. I. A. Visual sensitivity. Annual Review of Psychology, 1978, 29, 613-645. 
Marks, L. E. Sensory processes: The new psychophysics. New York: Academic Press, 1974.

Mueller, C. G. Frequency of seeing functions for intensity discrimination at various levels of adapting intensity. Journal of General Physiology, 1951, 34, 463-474.

STiles, W. S. Color vision: The approach through incrementthreshold sensitivity. Proceedings of the National Academy of Sciences, 1959, 45, 100-114.

UTTAL, W. R. The psychobiology of sensory coding. New York: Harper \& Row, 1973.

Wasserman, G. S. Unconditioned response to light in Limulus: Mediation by lateral, median, and ventral eye loci. Vision Research, 1973, 13, 95-105.

Wasserman, G. S. Limulus psychophysics: Dark adaptation in the ventral eye. Journal of Experimental Psychology: Human Pereeption and Performance, 1975, 104, 68-76.

Wasserman, G. S. Limulus psychophysics: Spectral sensitivity of the ventral eye. Journal of Experimental Psychology: General, $1976,105,240-253$.

Wasserman, G. S. Limulus psychophysics: Temporal summation in the ventral eye. Journal of Experimental Psychology: General, $1978,107,276-286$.

Wasserman, G. S., \& Kong, K.-L. Illusory correlation of brightness enhancement and transients in the nervous system. Science, 1974, 184, 911-913.

Wasserman, G. S., \& Kong, K.-L. Temporal summation in a photoreceptor: Dependence on response magnitude. Vision Research, 1975, 15, 1297-1299.

Wasserman, G. S., \& Patton, D. G. Limulus visual threshold obtained from light-elicited unconditioned tail movements. Journal of Comparative and Physiological Psychology, 1970, 73, $111-116$.

WULFF, V. J. Duality in the electrical response of the lateral eye of Limulus polyphemus. Biological Bulletin, 1950, 98, 258-265.

\section{NOTES}

1. Comparisons made by Clack and Pepperberg (Note 2).

2. Spectrum provided by Barlow (Note 3 ).

3. The relation between an inflected intensity-response function and an inflected increment threshold function can best be described with the aid of Figure 7. This is a plot of the receptor re-

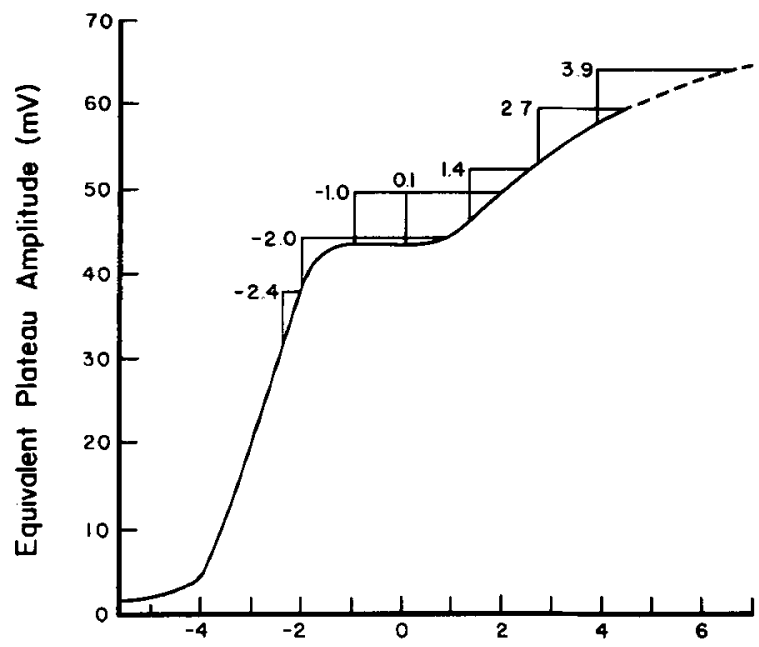

Log Intensity $\left(\mathrm{mW} / \mathrm{m}^{2}\right)$

Figure 7. Schematic representation of the calculations that relate the intensity-response function of a photoreceptor to the behavioral increment threshold function. See Footnote 3 for explanation. sponse integral as a function of intensity. The solid portion of the curve is taken from Bayer and Barlow's Figure 6. The dashed portion represents an extrapolation of their data. The response units are given in terms of the equivalent amplitude of the plateau, but the actual measurement was of the integral of the response cumulated over a 5-sec period, which commenced $5 \mathrm{sec}$ after stimulus onset. Data from other sources (Wasserman, Felsten, \& Easland, 1979) show that the integral of the initial transient portion of the receptor response generally follows the same inflected pattern. Hence, these results are unlikely to have been markedly distorted by omitting the initial portion of the receptor response.

One can construct a behavioral increment threshold function from the receptor intensity-response function shown in Figure 7 using Fechner's scaling assumption. The staircase-shaped steps superimposed upon the intensity-response function illustrate this calculation: One determines the test-flash threshold on any given background that would correspond to a criterion increment of the sensory signal by locating the intensity that gives a response that is that criterion amount larger than the response given by the background. The staircase step (labeled -2.4 in the figure) shows this construction for a background intensity of $-2.4 \log$ units. The other staircase steps in the figures show the same construction for other backgrounds. The inflected character of the resulting increment threshold function is an immediate result of these constructions: At low background intensities, the threshold intensity is only slightly higher than the background because here the intensityresponse function is very steep. But when the intensity-response function levels off at intermediate intensities, the test-flash threshold rises very rapidly. Then, when several background intensities all fall on the flat portion of the intensity-response function, the testflash threshold remains invariant. Thus, a background of $-1 \log$ unit and a background of $.1 \mathrm{log}$ units both give the same testflash threshold because both backgrounds produce the same response. When the intensity-response function again begins to rise, the background intensities again increase, but at a less rapid rate.

Essentially the same analysis can be run in reverse if one starts from behavioral measurements of the increment threshold. Each background intensity used in an increment threshold experiment can be used to calculate the corresponding response amplitude. Similarly, each test-flash threshold can be used to calculate its corresponding response amplitude. The response-amplitude difference between a given background and its corresponding testflash threshold provides one estimate of the size of the incremental response needed.

If Fechner's scaling assumption (that equally detectable light increments are mediated by equal neural increments) were correct, then these estimates of the neural increments calculated from behavior would all have the same magnitude. This outcome was not obtained when Bayer and Barlow's intensity-response function was used without any calibration adjustment. Instead, the response pair differences were too large at low backgrounds and too small at high backgrounds. In order to satisfy the equality requirement of Fechner's scaling assumption, it was necessary to shift Bayer and Barlow's intensity-response curve to the left by a factor of $1.8 \mathrm{log}$ units to fit the fall data and by a factor of $1.3 \mathrm{log}$ units to fit the winter data. (The curve shown in Figure 7 represents a sensitivity shift of $1.3 \mathrm{log}$ units.) When these sensitivity shifts were made, the quality of the theoretical account was excellent, and the absolute values of the residuals averaged $7.4 \%$ of the mean difference in the fall and $15.6 \%$ in the winter. The greatest discrepancy occurred in each season at the highest background intensity. As noted above, the calculations at these intensities were based upon an extrapolation of Bayer and Barlow's sensitivity function, which assumed that the response gradually saturates. This assumption may be incorrect. The theoretical curves in Figure 4 were then calculated by using the shifted intensity response and a sensory criterion taken as the average of the behavioral response pair difference. This calculation has one free parameter, the shift value. Figure 7 illustrates the actual calculations for the winter experiment. 
Some sensitivity shift was not unexpected because there is some uncertainty about the absolute absorption of the ventral eye cuticle (Wasserman, 1976). But, it seems unlikely that the cuticle absorption estimate cóld have been off by either 1.3 or $1.8 \mathrm{log}$ units because the cuticle correction was only $1.1 \log$ units. In order to explain these sensitivity adjustments on cuticle grounds, the cuticle would have to have a negative absorption of either .2 or $.7 \mathrm{log}$ units. Either outcome is impossible. One possible explanation of the sensitivity adjustment is that the behavior is based on the average of signals coming from many receptors, whereas Bayer and Barlow's intensity-response function was taken from only one cell. The intensity at which the inflection falls in the collection of cells studied by Barlow and Kaplan and by Bayer and Barlow varied over a range of about $2 \mathrm{log}$ units. Such variation is not uncommon in single-cell research. An alternative possibility is that any physiological measurement interferes with the system and reduces its sensitivity. On either assumption, the behavioral measures would be the more precise estimates of sensitivity because they would be less subject to both random and systematic effects.
4. These values are relatively insensitive to the sensitivity adjustment described above. Had no adjustment been made, they would have been about $4 \mathrm{mV}$ larger.

5. This fit also required a sensitivity adjustment of the physiological data. Because Bayer and Barlow (1978) did not report variabilities, the variability calculation had to be taken from a cell (in the lateral eye) different from the one (in the ventral eye) used to predict the increment threshold function; the sensitivity of the former differed from that of the latter by about $1 \log$ unit. To equate the sensitivities used for both the mean calculations and the variability calculations, the receptor variability data were therefore shifted by an amount that was 1 log unit less-namely, .3 log units in winter and .8 in fall. The fact that an equivalent sensitivity shift accounts for the variabilities as well as the means reinforces the validity of the notion that the cause of the adjustment is the intrinsic cell-to-cell variability characteristic of most single-cell research.

(Received for publication March 20, 1980; revision accepted November 26, 1980.) 